\title{
I.
}

Aus dem St. Johannishospital zu Bonn (Chirurgische Abteilung, Chefarzt weil. Herr Geheimrat Prof. Dr. Schede).

\section{Zur Therapie der Sarkome der langen Röhrenknochen.}

\author{
Von \\ Dr. Karl Vogel, \\ Sekundürarzt, Privatdozent für Chirurgie.
}

Wenn wir heute die Veröffentlichungen der letzten Jahrzehnte betr. die Therapie der Sarkome der langen Röhrenknochen überblicken und die Grundsätze, die für dieselbe bei den Chirurgen maßgebend geworden sind, so finden wir, daß so ziemlich allseitige Übereinstimmung nur herrscht in bezug auf eine Form der Sarkome, nämlich die sog. "schaligen Riesenzellensarkome". Es sind das diejenigen Tumoren, die myelogen entstehend, langsam wachsend, den Knochen an der betroffenen Stelle kolbig auftreiben, die Kortikalis bis zu Papierdünne rarefizieren, dabei aber fast stets zirkumskript, gegen die gesunde Umgebung gut abgesetzt bleiben. Sie brechen erst spät in die Weichteile durch, machen fast nie Metastasen, selten Rezidive.

Bei dieser Form begnügt sich die heutige Chirurgie meist mit dem möglichst konservativen Eingriff. Man legt den Tumor durch Aufmeißeln der Knochenschale frei und evidiert alle Massen mit dem scharfen Löffel. Event. kann man das Lager noch verschorfen oder verätzen. Etwaige lokale Rezidive werden ebenso behandelt. In der Literatur sind recht viele Fälle niedergelegt, in denen diese sehr schonende Methode entweder nach einmaliger Operation oder doch nach Entfernung 'eines oder mehrerer kleiner Rezidive zur Dauerheilung geführt hat. Im vorigen Jahr ist aus unserem Hospital ein hierher gehöriger Fall von Schmieden veröffentlicht worden. Dic Patientin ist heute noch, nach fast drei Jahren, rezidivfrei. Krause berichtete auf dem Chirurgenkongress 1889 über einen Fall, in dem v. Volkmann 4 mal einen derartigen Tumor des Unterkiefers evidiert Deutsche Zeitschrift f. Chirurgie. LXX. Bd. 
hatte mit endlichem Dauererfolg. Derselbe Operateur hat einen gleichen Tumor der Tibia ebenso konservativ behandelt, der zur Zeit des Berichtes seit $2^{3 / 4}$ Jahren rezidivfrei war.

Diese Beispiele mögen für diese Form der sog. benignen Sarkome genügen. Eine erschöpfende Übersicht der hierher gehörigen Fälle zu geben, würde wenig Zweck haben, da, wie gesagt, über die Berechtigung, derartige Tumoren durchaus konservativ zu behandeln, so ziemlich Einigkeit herrscht.

Es finden sich nun aber auch einige Fälle in der Literatur, in denen ein pathologisch-anatomisch diesen Riesenzellensarkomen analoger Tumor klinisch sofort oder nach einiger Zeit, etwa nach einer ersten konservativen Exstirpation, den gutartigen Charakter verlor, indem er den Knochen durchbrach, in die Nachbarschaft propagierte und durch Metastasenbildung sich als evident bösartig erwies. Gross, Nasse u. A. erwähnen solche Fälle. Einen besonders eklatanten Fall schildert Oberst. Es hat sich daher die Praxis herausgebildet, bei der Operation dieser Tumoren sich weniger durch den pathologisch-anatomischen, als durch den klinischen Befund leiten zu lassen. Ist der Tumor zirkumskript, ein eigentliches "schaliges" Riesenzellensarkom, so kann man sich zunächst mit oben geschilderter konservativer 'Therapie begnügen, hat er jedoch die Grenze des Knochens überschritten und die Weichteile ergriffen, so gehört er für den Operateur zu den eigentlichen malignen Tumoren, die stets radikaler angegriffen werden müssen, ohne Rücksicht auf den histologischen Befund.

Ich komme damit auf die Therapie der eigentlich bösartigen Sarkome, der kleinzelligen Rund- und Spindelzellensarkome. Sie sind vielleicht dasjenige Leiden, dessen Prognose durch alle Fortschritte sowohl der pathologischen Anatomie als der chirurgischen Technik bis jetzt am wenigsten hat verbessert werden können. Die Mortalitätsstatistik der Sarkome zeigt heute noch die Ohnmacht ärztlicher Kunst in betriibender Weise.

Kramer hat jüngst in "Langenbecks Archiv" die chirurgische Therapie der bösartigen Sarkome der langen Röhrenknochen besprochen und kommt zu dem Schluß, daß eine Dauerheilung derselben nur dann möglich sei, wenn der Tumor abgekapselt sei, daß aber bei Übergreifen des Tumors auf die benachbarten Weichteile auch von Exarticulation und Amputation der Extremität selten etwas zu erhoffen sei. Es sei in solehen Fällen außer der Absetzung des Gliedes unbedingt nötig, die ergriffenen Muskeln in toto zu entfernen.

Auch Schuchardt rät möglichst gridndliche Exartikulation und Amputation inklusive der zugehörigen Muskelbäuche, stellt aber auch dann 
noch die Prognose quoad Rezidiv und Metastase schlecht. Reinhardt warnt vor partieller Operation bei anderen als Riesenzellensarkomen, weil außer dem Tumor noch zentralwärts isolierte Knoten im Schaft des Knochens vorkommen, welche Tatsache anch König hervorhebt. Reinhardt rechnet aus 54 zusammengestellten Fällen 18 Proz. Dauerheilung heraus (itber 4 Jahre).

In ähnlicher Weise sprechen sich die meisten Autoren aus und so findet man denn bei Durchsicht der in der Literatur niedergelegten Fälle, daß bei weitem die überwiegende Anzahl aller Extremitätensarkome mit sofortiger Absetzung des Gliedes behandelt worden sind.

Vor einigen Jahren hat nun $\mathrm{v}$. Mikulicz an Stelle der prinzipiellen sofortigen Exartikulation und Amputation die natülich auf geeignete Fälle zu beschränkende Resektion des Tumors und seiner nächsten Umgebung bis ins gesunde Gewebe aus der Kontinuität, also mit Erhaltung des peripherwärts vom Tumor gelegenen Gliedteils empfohlen und diesen Rat durch sehr beweiskräftige Argumente gestützt.

Er erinnert zunächst an einige in den letzten Jahren vor Erscheinen seiner Arbeit veröffentlichte Fälle von Resektion: Auf der Naturforscherversammlung in Halle stellte v. Bergmann einen Patienten vor, bei dem er wegen periostalen Tibiasarkoms ein $15 \mathrm{~cm}$ langes Stück dieses Knochens und nachher ein entsprechendes der Fibula reseziert hatte. Gute Heilung. Ein entsprechend erhöhter Schuh verschaffte dem Patienten eine recht gute Gebrauchsfähigkeit des operierten Beines. Ein zweiter Fall stammt aus der v. Bramannschen Klinik: Wegen myelogenen Sarkoms des linken Tibiakopfes wurden $10 \mathrm{~cm}$ rom Kniegelenk aus reseziert. Die Gebrauchsfähigkeit des Beines wurde dadurch erreicht, daß von der Femurgelenkfläche eine Scheibe abgeschnitten, an der fibularen Seite jener ein Bohrloch im Femur angebracht und in dasselbe die abgesägte und zugespitzte Fibula so tief eingepflanzt wurde, daß die Sägeflächen von Femur und Tibia sich beruhrten. Knöcherne Verheilung und gute Funktion des mit mäßiger Verkürzung ankylotischen Beines unter Anwendung eines erhöhten Schuhes, ohne weitere Protese. V. Mikulicz erwähnt, daß dieser Patient noch nach drei Jahren rezidivfrei war.

An der oberen Extremität hat v. Mikulic z mehrere Resektionen teils noch aus früherer Zeit gefunden: So eine solche der unteren Hälfte der UIna wegen Myeloidsarkom mit gutem funktionellem Resultat, ausgefuthrt von Lucas. Morris entfernte sogar die unteren $2 / 3$ des Radius und 3 bis 4 Zoll der Ulna wegen gleichen Tumors. Hier wurde zur Stätze der Hand ein Lederapparat gegeben, der relativ gute Funktion ermöglichte. Ein dritter ähnlicher Fall stammt von Berkeley Hill.

Endlich zitiert v. Mikulicz noch zwei ältere Fälle von Volkmann, in deren einem dieser das obere Humerusende wegen Spindelzellensarkoms resezierte; Patient starb nach 7 Tagen an Kollaps. Im anderen Falle entfernte v. Volkmann das untere Ende des Humerus und das Olekranon wegen Enchondroma myxomatodes mit guter Heilung und ziemlich festem Gelenk: Letzterer Fall ist nur einige Monate beobachtet worden.

An diese kasuistische Besprechnng schliesst v. Mikulicz die Schilderung seiner eigenen interessanten Fälle, die ich in kurzem Auszuge zitieren möchte. 
In den beiden ersten Fällen handelt es sich um Riesenzellensarkome des Radius, durchgebrochen in die Weichtheile im 1., noch mehr zirkumskript im 2. Falle. In jenem wurde von einem radialen Längsschnitt aus $12 \mathrm{~cm}$ des Radius mit den ergriffenen Weichteilen entfernt. Streckund Beugesehnen werden mit Ausnahme des Supinator longus und Pronator quadratus erhalten, Art. und Nerv. radialis geopfert. Glatte Heilung; gute Funktion mit Lederhulse. Nach 11/2 Jahren kein Rezidiv. Im zweiten Falle, einem nach Trauma entstandenen Tumor, werden vom gleichen Schnitt ans $10 \mathrm{~cm}$ Radius vom Handgelenk aus entfernt. Glatte Heilung. Patient wird mit Lederbulse wieder voll erwerbsfähig. Rezidivfreie Beobachtungszeit 4 Monate. Es folgt ein Fall von Resectio ulnae wegen mit den Weichteilen verwachsenem Spindelzellensarkom. Die ergriffenen Weichteile werden mit entfernt, von der Ulna das Mittelstück so reseziert, daß oben 6 , unten $4 \mathrm{~cm}$ stehen bleiben. Kleine Sehnenplastik, Entfernung mebrerer Kubital- und Achseldrüsen; Heilung; weiteres Schicksal unbekannt. Im 4. Falle werden von vorderem Lappenschnitt aus $10 \mathrm{~cm}$ der Tibia vom Kniegelenk an reseziert wegen abgekapselten Riesenzellensarkoms. Absägen der Femurgelenkfläche und der Fibula in der Höhe des Tibiaschnittes, Interposition des zentralen mit den Weichteilen in Verbindung gebliebenem Fibulastttckes zwischen Tibiarest und Femur, in welche jenes in je ein Bohrloch eingepflanzt wird. Heilung ziemlich gut, doch erfullt sich die Hoffnung, daß das implantierte Fibulastück funktionell soweit hypertrophieren werde, daß ein knöchern ankylotisches und tragfähiges Bein resultieren werde, nicht. Patient muss einen fixierenden Verband tragen. Über sein weiteres Schicksal ist nichts bekannt.

Endlich gehören hierhin zwei Fälle von Resectio femoris, einmal wegen diffus in die Muskulatur vorgedrungenem Spindelzellen-, das andere Mal wegen Spindelrundzellensarkoms. Im ersteren Falle wurden von einem vorderen viereckigen nach oben umgeklappten Lappenschnitt aus, der an der Basis noch etwas gesunde Quadrizepsmuskulatur enthielt, sonst ans Haut und Unterhautfettgewebe bestand, $20 \mathrm{~cm}$ des Femur entfernt, nach Freipräparieren der Arteria und Vena popl., sowie des $\mathrm{N}$. ischiadicus der Femurstumpt in ein Bohrloch der von der Knorpelfäche befreiten Tibia implantiert. Nach glatter Heilung wird die $20 \mathrm{~cm}$ betragende Verkürzung durch eine sinnreiche Protese ausgeglichen. Nach $2^{1 / 2}$ Monaten ist Patient noch gesund.

Der Befund und die Operation des folgenden Falles war ähnlich; Entfernung der untersten $20 \mathrm{~cm}$ des Femur, zwischen dem Stumpf und die angefrisehte und mit einem Bohrloch versehene Tibia wurde hier ein Elfenbeinrohr eingeschaltet. Die Wundheilung erfolgt ohne Störung, doch tritt keine Konsolidation ein und wird nach 5 Monaten wegen dieses Umstandes und gleichzeitigen Verdachtes auf Rezidiv nachträglich die Amputation vorgenommen. Es findet sich jedoch kein Rezidiv.

In einem letzten Falle hat v. Mikulicz bei analogem Befund dieselbe Operation gemacht, aber wegen Verwachsenseins des Tumors (periostales Osteosarkom) mit den Gefäßen die sofortige Amputation angeschlossen. Endlich erwähnt v. Mikulicz in einer Anmerkung noch einen in jüngster Zeit operierten, dem oben zitierten v. Bramannschen ganz analogen Fall mit glatter Heilung. 
Außer diesen Fällen von Resektion habe ich in der jüngeren Literatur noch folgende gefundell: Nasse schildert einen Fall von Spindelzellensarkom des unteren Femurendes, im Knochen gut isoliert, aber gegen die Narbe und Fistel von einer vorher anderwärts gemachten Inzision nicht dentlich abgesetzt. (Fall 8 der Tabelle.) Das untere Femurende wurde reseziert und die verdächtigen Weichteile exzidiert. Heilung; Patient ist seit drei Jahren gesund.

Außerdem ist in Fall 3 der $\mathrm{N}$ asseschen Tabelle wegen eines Sarkoms des unteren Femurendes, dessen pathologisch-anatomische Diagnose nicht angegeben ist, die Resectio genu gemacht worden; doch mußte nach 6 Monaten wegen Rezidiv die Amputation ausgefubrt werden.

Endlich wurde in Fall 4 wegen Riesenzellensarkoms die untere Partie der Tibia reseziert. Die Patientin ist 5 Jahre gesund.

Rotter hat 1898 in der "Freien Vereinigung der Chirurgen Berlins" einen Patienten vorgestellt, dem er den ganzen Humerus wegen Sarkoms exstirpiert hatte. Der N. radialis wurde zu $2 / 3$ reseziert. Der Patient bekam eine relativ recht gute Funktion der Hand. R. hatte hier die konservative Operationsmethode gewählt wegen einer angenommenen relativen Benignität des Tumors, in dessen Struktur das fibröse Gewebe tuber die zelligen Elemente überwog.

Wiesinger veröffentlicht drei Fälle von Resektion aus dem Hamburger alten Krankenhaus. Einmal resezierte er das obere Drittel des Humerus wegen Rund- und Spindelzellensarkom; Heilung; nach 1/2 Jahr noch gesund.

Im zweiten Fall wurde wegen periostalen Osteochondrosarkom der untere Femurteil reseziert und der Stumpf in die Tibia eingepflanzt; Heilung; nach $1 / 2$ Jahr noch gesund.

Endlich entfernte er im dritten Falle das obere Tibiaende wegen Chondrosarkoms und heftete auch hier Tibia und Femur aneinander.

$R$ heinwald beschreibt einen Fall, in dem ein zweifaustgroßes Sarkom des rechten Oberarmes entfernt wurde. Nach 5/4 Jahren bekam Patient an symmetrischer Stelle des linken Armes einen ebensolchen Tumor. Hier wurde die Resektion des oberen Humerusendes gemacht, doch starb Patient schon nach 8 Monaten an Lebermetastasen.

Endlich hat K örte 1901 einen Patienten demonstriert, dem er $19 \mathrm{~cm}$ des Femur resezierte wegen periostalen Spindelzellensarkoms. Ausgang in Heilung. Beobachtungszeit noch kurz.

Wegen schaliger Riesenzellensarkome, also relativ gutartigen Tumors, haben noch analoge Resektionen ausgeführt Eve und Clutton.

An diese kasuistische Zusammenstellung möchte ich die Krankengeschichte eines Patienten anschließen, der vor jetzt gerade 4 Jahren hier von Herrn Geheimrat Schede operiert worden ist und sich bis heute wohl befindet.

Otto H. aus W., 34 Jahre alt, Kaufmann; aufgenommen 19. Juli, entlassen 15. August 1899.

A namnese: Patient war fruher gesund. Vor zwei Jahren hat er zuerst das Auftreten einer Geschwulst in der Gegend des linken Schultergelenkes bemerkt, die, „von selbst“ entstanden, ziemlich schnell gewachsen 
ist. Vor $1^{1 / 2}$ Jaliren wurde der Tumor auswärts zum erstenmal operiert und zwar wurden damals schon die distalen Gelenkenden der Clavicula und Scapula und das proximale des Humerus, von welch letzterem die Geschwulst ausgegangen sein soll, mitentfernt. Nähere Angaben sind uber die damalige Operation nicht zu bekommen. Patient war dann zunäclsst gesund, hat aber seit einigen Monaten wieder eine Anschwellung bemerkt, die ohne Schmerzen sich vergrösserte und die geringe nach der ersten Operation vorhandene Beweglichkeit des Armes noch mehr beschränkte.

Status: Großfaustgroßer, ziemlich derber Tumor nuter, respektiv im Musc. deltoideus links, nicht verschieblich. Beweglichkeit des Armes gering. Keine Schmerzen; Haut intakt. Senkrechte Narbe über dem Sulcus bicipitalis $15 \mathrm{~cm}$ lang.

Diagn ose: Sarkomrezidiv.

Operation: Hautschnitt senkrecht ther die Höhe der Geschwulst. Der Tumor sitzt unter dem Musc. deltoideus. Letzterer wird nach beiden Seiten abpräpariert. Der Tumor enthält einige Cysten mit gallertigem Inhalt. Er ist mit dem Akromion und dem distalen Stumpf der Clavicula verwachsen, ebenso mit dem zentralen Humerusstumpfe. Alle drei Knochenenden sind in ihrer Struktur zerstört und gehen direkt in die weichere Tumormasse über. Letztere geht auch stellenweise in die Muskulatur der Schnltergegend über. Durchmeißeln respektiv -sägen der Clavicula und Skapula im Gesunden, Freipräparieren des oberen Humerus und Absägen des letzteren $4 \mathrm{~cm}$ unterhalb der sichtbaren Geschwulstgrenze. Auslösen des Tumors aus den Weichteilen unter Wegnahme des grössten Teiles der erkrankten Muskulatur, das heißt, soweit es klinisch erkennbar war, therall im Gesunden. Es zeigte sich beim Durchsägen des Humerus, daß die Markhöhle desselben an der Sägestelle und distalwärts darüber hinaus ganz mit Tumormasse ausgefüllt ist. Ein Sägeschnitt einige Centimeter abwärts zeigt noch dasselbe Bild und so wird in mehreren Sttleken der ganze vorher skelettierte Humerus entfernt, mit Ausnahme der untersten $3 \mathrm{~cm}$, also des Gelenkendes des Ellenbogens, nachdem $3 \mathrm{~cm}$ oberhalb das Mark makroskopisch gesiund befunden worden war. Die Gefäße und Nerven waren nicht ergriffen und wurden geschont. Die große Wunde wird zunächst tamponiert, mit Situationsuähten versehen und der Arm mit Schienen und vielen Kissen verbunden.

1. August. Sekundärnaht der gut granulierenden Wunde durch tiefgreifende Nähte.

Glatte Heilung. Patient wird mit einer an Schulter und Brust zu befestigenden Lederhülse für den Oberarm und einen Teil des Unterarmes mit Charniergelenk am Ellenbogen entlassen. Nach einiger Gewöhnung kann er den Arm und besonders die Hand recht gut gebrauchen.

Ich habe den Kranken nicht wieder gesehen, weiß jedoch aus wiederholten schriftlichen Berichten, noch aus jungster Zeit, daß es ibm durchaus gut geht und er seinem Berufe ungestört nachgeht.

Pathol.-a a tomische Diagn ose (Pathol. Institut der Universität): Rundzellensarkom vom Charakter des Chondromyxosarkoms.

Wir haben also einen sowohl pathologisch-anatomisch, wie klinisch durchaus malignen Tumor, der schon einmal recht radikal exstir- 
piert worden und jetzt wieder in schnellem Wachstum begriffen ist, und der trotzdem durch die konservative Methode der Resektion geheilt wird. Denn daß unser Patient jetzt definitiv geheilt ist, können wir wohl annehmen, nachdem 4 Jahre olne Rezidiv und Metastase verflossen sind.

Bei weitem die meisten Metastasen nach Sarkomen treten bald nach der Operation auf, etwa im ersten Jahr. Im zweiten Jahre sind sie recht selten; nach dem zweiten Jahre kommen sie nur noch sehr rereinzelt zur Beobachtung vor. Sie müßten, wie Nasse mit Recht sagt, vor Entfernung des Tumors schon vorhanden gewesen sein, könnten also nur bei ganz langsam wachsenden Tumoren so spät erst in die Erscheinung treten.

Etwas ungünstiger liegen die Verhältnisse in bezug auf die lokalen Rezidive. Sie treten auch meist bald nach der Exstirpation des Tumors auf, jedoch sind einzelne Fälle, so durch Nasse u. A. bekannt geworden, in denen selbst sehr schnell gewachsene primäre Sarkome nach der Exstirpation noch sehr spät, nach vier Jahren und später, Rezidive im Gefolge gehabt haben. Doch sind diese Fälle so äußerst selten, daß sie, wie auch $\mathrm{N}$ as se sagt, um eine Statistik zu ermöglichen, außer acht gelassen werden können. Der letztere Autor resultiert aus seinen Beobachtungen, daß derjenige Patient als geheilt zu betrachten ist, der zwei Jalıre nach der Operation noch gesund ist. Wir können demnach unseren Patienten jetzt nach 4 Jahren sicher als dauernd geheilt betrachten.

Es liegt mir nun zwar fern, aus diesem vereinzelten Falle den Schluss zu ziehen, daf man in Zukunft alle ähnlichen Fälle mit konservativer Resektion behandeln solle. Der günstige Ausgang ist hier sicher fast ein Unikum. Aber wenn v. Mikulicz sagt, er möchte glauben, daß man die konservativen Methoden bei Knochensarkomen mehr als bisher anwenden könne und man dürfe es wenigstens nicht unversucht lassen, „der konservativen Chirurgie hier ein größeres Feld zu erobern," so möchte ich unseren Fall für 'diesen Rat doch als Stütze dienen lassen, indem er, so vereinzelt er ist, die Möglichkeit des Erfolges doch beweist. Wenn ich diesen Fall mit den oben zitierten aus der Literatur zusammenstelle und auch zur Beurteilung der Prognose diejenigen ausnchme, in denen es sich um relativ gutartige Riesenzellensarkome handelte und die ich mehr zur Illustration der angewendeten Technik angeführt habe, so scheint mir doch das Gesamtresultat einer Betrachtung dieser Fälle wenigstens durchaus nicht gegen jenen Mikuliczschen Vorschlag zu sprechen, die Resektion etwas öfter als es bisher geschehen zux Entfernung derartiger Tumoren 
in Erwägung zu ziehen. So ist doch sehr zu berücksichtigen, daß auch die Prognose der Radikaloperation, der Exartikulation und Amputation, sehr schlecht ist. Ein Vergleich der Erfolge beider Methoden, der allein entscheidend wäre, läßt sich leider nicht wohl anstellen, da die weitaus meisten operierten Patienten entweder nicht bis zum Tode oder dem Ende einer "kritischen Zeit" beobachtet oder die Resultate der Operation in bezug auf Dauerheilung nicht in der Literatur mitgeteilt sind. Zudem ist das Mißverhältnis der Zahlen der ausgeführten Amputationen und Exartikulationen einerseits und der Resektionen andererseits ein zu groles, die absolute Zahl der letzteren zu klein, als daß ein maßgebender Vergleich möglich wäre. Es gibt jedoch eine Anzahl Gründe, die einer häufigeren Anwendung der Resektion das Wort reden.

Körte verteidigt das in obigem Fall von Resektion bei Spindelzellensarkom angewandte Verfahren gegen den Vorwurf Königs, daß weiter oben in der Markhöhle noch Herde zurückbleiben könnten, dadurch, daß er auf die doch auch ungünstige Prognose der Radikalverfahren hinweist, besonders was die Metastasen betrifft. Ich möchte auch glauben, daß es in bezug auf die letzteren vollkommen gleichgültig ist, ob man amputiert oder reseziert. Entweder die Aussaat in den übrigen Körper hat vorher schon stattgefunden, dann wird man mit keiner Methode etwas erreichen; oder der Prozel ist noch lokal, dann wird nach der Resektion wohl die Prognose in bezug auf Metastasen sich nicht schlechter gestalten als nach der Amputation. Lokale Rezidive dagegen wird zweifellos unter Umständen das radikale Verfahren der Absetzung des ganzen Gliedes besser verhüten, als die Resektion, da abseits gelegene Knochenherde, wie sie König und Reinhardt erwähnen, oder auch solche der Weichteile bei jenem sicherer mit entfernt werden.

Körte sagte, man könne ja beim Auftreten von Rezidiven immer noch die Entfernung der Extremität ausführen. Das kann wohl nur bedingungsweise als Rechtfertigung der Resektion verwandt werden. Bei sehr sorgfältiger Beobachtung der Patienten und wenn das Rezidiv an einer der Wahrnehmung gut erreichbaren Stelle auftritt, etwa unter der Haut oder an den Drüsen, wird man wobl meist die sekundäre Amputation unter nicht schlechteren Bedingungen für den Patienten machen, als wenn man sie direkt bei der ersten Geschwulst ausgeführt hätte. Tritt jedoch das Rezidiv an einer Stelle auf, die es erst später in die Erscheinung treten läßt, wenn es schon länger besteht und eine größere Ausdehnung erreicht hat, oder läßt man gar bei der ersten Operation einen der obenerwähnten Herde in der 
Nachbarschaft zurück, so muß man doch zugeben, daß leicht zwischen erster und zweiter Operation resp. während !des Wachstums des Rezidivs bis zu seiner Entfernung eine Aussaat von Keimen in den übrigen Organismus erfolgen kann, die man bei primärer Radikaloperation vermieden hätte.

Zudem ist bekannt, daß gerade bei den malignen Tumoren eine Operation, wenn sie nicht alles Kranke entfernt, oft $\mathrm{zu}$ schnellerem Wachstum und zu intensiverer Äusserung des malignen Charakters (schnelleres Auftreten der Metastasen) anregt. Vielfach wird auch doch eine sekundäre Amputation oder Exartikulation unter ungünstigeren Allgemeinverhältnissen stattfinden und dadurch die Prognose in bezug. auf die direkte Operationsmortalität verschlechtert werden, worauf u. a. Berger hingewiesen hat. Eine Exartikulation ist schon an sich kein so kleiner Eingriff, besonders, wenn man die Forderung Kramers und anderer erfüllt, die Muskulatur der Schulter resp. Hüfte soweit als möglich mitzunehmen. Wenn der Eingriff an der Schulter auch bei der hentigen Technik nicht so direkt lebensgefährlich ist, so ist speziell eine Hüftexartikulation mit Wegnahme der Muskeln immer ein Eingriff von zweifelhafter Prognose, trotz der wesentlichen Verbesserung und Herabminderung der Gefahr, besonders des Blutverlustes, die durch das $\mathbf{R}$ os esche Verfahren bedingt wird.

Ein nicht hoch genug zu veranschlagendes psychologisches Argument für die Resektion hebt v. Mikulicz hervor: Ein Kranker wird sich viel eher zu einer Resektion, wobei ihm das Glied, wenn auch funktionell minderwertig, erhalten bleibt, entschließen, als zu einer sofortigen Entfernung desselben. Diejenigen Patienten, die schon mit großen Tumoren in chirurgische Behandlung kamen, geben ja eher zur Amputation die Erlaubnis, weil sie durch die Größe der Geschwulst, eventuelle Ulzerationen, Schmerzen und Gebrauchsunfähig. keit ängstlich geworden sind und die Notwendigkeit einer Radikaloperation einsehen. Diese Fälle geben aber bei jeder Operationsmethode eine scblechte Prognose. Gerade die Patienten aber, die bei sachgemäßer und vor allem frühzeitiger Behandlung gerettet werden können, das heißt die mit noch in den Anfängen der Entwicklung befindlichen Tumoren in die Behandlung kommen, sind naturgemäß am schwersten zur sofortigen Entfernung des ganzen Gliedes zu bewegen. Hier bestehen meist keine Schmerzen, wenig Funktionsstörung, der Patient sieht keine wesentliche Veränderung an seinem Arm oder Bein, und er vermag nicht einzusehen, weshalb er wegen eines in seinen Augen geringfügigen Leidens zum einarmigen oder einbeinigen Krüppel gemacht werden soll. 
Der Vorschlag zur Radikaloperation wird ihn, wie Mikulicz treffend sagt, in vielen Fällen zum Kurpfuscher treiben und ihn sachgemäße Hilfe erst dann wieder aufsuchen lassen, wenn es zu spät zur Operation überhaupt oder jedenfalls die Prognose auch für die radikalste Methode wesentlich verschlechtert ist. Die Resektion hätte in solchen Fällen frühzeitig gemacht werden können, als der Tumor noch klein, Metastasenbildung noch unwahrscheinlich war, die Exartikulation oder Amputation kommt zu spät. Das Prinzip also, diese Tumoren müßten unter allen Umständen durch Amputation geheilt werden, verschuldet hier sicher eine Verschlechterung der Gesamtprognose der Sarkombehandlung. Entsteht nach der primären Resektion ein Rezidiv, so wird der Patient sich weit eher zur Entfernung des Gliedes bestimmen lassen, die bei guter Beobachtung wohl meist zeitig genug ausgeführt werden kann.

Noch einen Punkt möchte ich hier im Anschluß an unseren Fall besprechen, den ich nirgends so recht gewürdigt gefunden habe. Ich meine nämlich, je proximaler ein Tumor sitzt, desto eher kann man sich zur Resektion anstatt der Exartikulation oder Amputation entschließen. Das klingt vielleicht paradox, doch möchte ich folgende Begründung geben: Sitzt ein Tumor am distalen Radius- oder Ulnaende, so wird man durch Exartikulation im Schultergelenk sehr weit im Gesunden operieren und dadurch die Garantien der radikalen Entfernung erhöhen. Bei der Resektion jedoch bleibt in demselben Falle ein grosser Teil sowohl der Knochen als der Weichteile des Armes z entral vom Tumor stehen und die Möglichkeit, hier Geschwulstteile zurückzulassen (s. oben König), ist also groß. Dieser zentrale Rumpf ist es ja, an dem diese disseminierten, von Haupttumor ausgegangenen Herde sitzen und die Rezidive auftreten, während solche an dem peripher von der Resektionsstelle stehen gebliebenen Teile der Extremität wohl nur äußerst selten beobachtet worden sind. Sitzt dagegen eine Geschwulst in der Nähe der Schulter, so fallen die Chancen der Exartikulation gewaltig, denn die Operation nimmt nicht soviel gesundes Gewebe mit weg, da ja doch eine Operation über den Schultergürtel hinaus nicht weiter zentralwärts ausgedehnt werden kann. Die Chancen der Resektion dagegen bleiben absolut genommen dieselben, da sie hier gerade so radikal ausgeführt werden kann, wie am Ilandgelenk, sie steigen also im Verhältnis zu denen der Exartikulation. Wie gesagt, der bei Resektion an der Schulter stehenbleibende periphere Teil des Armes bedingt keine Erhöhung: der Gefahr, wohl aber der bei Resektion am Handgelenk stehenbleibende zentrale Teil. Ich meine also kurz: Je näher ein Tumor an 
die Grenze, die von Natur für die Operation gesteckt ist, Becken- und Schultergürtel, heranrückt, desto mehr nähert sich die Resektion, was ihre Wertigkeit als Radikaloperation betrifft, der Exartikulation und um so schwerer fallen dann ihre anderen Vorzüge gegenüber jener ins Gewicht, vor allem die größere Bereitwilligkeit des Patienten zur Operation und der grole sowohl funktionelle als auch kosmetische Vorteil, den eine auch nur teilweise erhaltene Extremität vor der besten Protese bietet. Das auf unseren Fall angewandt, haben wir uns damals, als der Patient den dringenden Wunsch äußerte, wenn eben möglich, ihm den Arm zu erhalten, gesagt: Wenn man den Arm auch mit dem Schultergürtel exartikulierte, so müßten doch Gewebsteile zurückbleiben, die gerade so nahe am Tumor gesessen haben, und über deren Freisein von kranken Keimen man also ebensowenig Gewißheit hatte, als bei einer Resektion im makroskopisch Gesunden. Man kann ja heute, wie v. Mikulicz mit Recht sagt, die Grenzen für die Ausdehnung der Resektion sehr weit stellen und letztere wird tatsächlich erst dann unmöglich, wenn die Hauptgefälie an der betr. Stelle ergriffen sind. Die Forderung, im Gesunden zu operieren, besteht natürlich auch bei der Resektion zu Recht, und falls die ernährenden Gefäle für den distalen Teil der Extremität irgendwie suspekt sind, fällt natürlich jede Möglichkeit einer konservativen Operation von vornherein weg. v. Mikulicz hebt auch schon die größere Bösartigkeit der periostalen 'Tumoren gegenüber den myelogenen in dieser Richtung hervor. Den einen oder anderen kranken Nerven kann man schon eher mit resezieren, wie das ja in dem Fall von Rotter (s. oben) geschehen ist, der ${ }^{2 / 3}$ des Radialis aus der Kontinuität entfernte mit gutem funktionellem Resultat.

Was die Gebrauchsfähigkeit der Extremität nach Resektion und Amputation betrifft, so sind die Verhältnisse an der oberen zweifellos der Reselktion günstiger als an der unteren. Der Arm hat nicht als feste Stütze zu dienen und selbst bei sehr ausgedehnter Wegnahme proximaler Knochenpartieen, wie sie z. B. unser Fall und der von Rotter zeigen, ist mit einem guten orthopädischen Apparat eine recht leidliche Funktion der distalen Teile der Extremität zu erzielen. Am Bein dagegen ist die conditio sine qua non für eine gute Funktion die Erzielung einer ausreichenden Stabilität vom Becken bis zum Fuß. Auf Nichterfüllung dieser Forderung beruht das schlechte funktionelle Resultat einiger Literaturfälle, z. B. Fall 4 und 6 von v. Mikulicz, und jedenfalls hat $\mathrm{Wi}$ iesinger Recht, wenn er sagt, daß eine gute Protese nach Amputation des Beines besser ist, als eine nicht in guter Konsolidation ausgeheilte resezierte Extremität. Bei der Frage der 
Erzielung dieser festen Konsolidation der Knochenfragmente spielt sicher, wie einige Autoren angeben, das Lebensalter der Patienten eine wichtige Rolle, indem die noch in lebhafterem Wachstum begriffenen oder doch zu ausgiebigerer regenerativer Tätigkeit fähigen Knochen jugendlicher Personen bessere Aussichten geben als die älterer Leute.

Ich glaube aus dieser Besprechung der einschlägigen Momente im Anschluß an unseren so günstig verlaufenen Fall eine Wiederholung des von v. Mikulicz ausgesprochenen Rates herleiten zu dürfen, für geeignet erscheinende Fälle mehr als bisher das konservative Verfahren in Erwägung zu ziehen und möchte annehmen, die hauptsächlichsten Punkte hervorgehoben zu haben, die bei der Auswahl der "geeigneten" Fälle zur Richtschnur dienen können.

\section{Literaturverzeichnis.}

1) Schmieden, Deutsche med. Wochenschr. 1900.

2) Krause, F., Chirurgenkongreß. 1889.

3) Gro \$, Americ. Journ. of the medic. sciences. 1879.

4) Nasse, D., Langenbecks Archiv. 39. Bd.

5) Oberst, Deutsche Zeitschr. f. Chirurgie. 1881. 14. Bd.

6) Kramer, Langenbecks Archiv. 66.

7) Schuchardt, Dentsche Chirargie. 28.

8) Reinhardt, Chirurgenkongreß. 1897 u. Deutsche Zeitschr. f. Chirurgie. 47.

9) König, Freie Vereinigung der Chirurgen Berlins. Sitzung v. 11. Febr. 1901.

10) Körte, Ebenda.

11) v. Mikulicz, Langenbecks Archiv. 50.

12) v. Bergmann, Zentralbl. f. Chirurgie. 91. 943.

13) v. Bramann (Neumann), Chirurgenkongreß. 1893 u. 1894.

14) v. Volkmann, Langenbecks Archiv. 15.

15) Rotter, Freie Vereinigung der Chirurgen Berlins. Sitzung vom Januar 1898.

16) Wiesinger, Deutsche med. Wochenschr. 1898, 42.

17) Rheinwald, Bruns' Beitr. 32 .

18) Berger, L'amputation du membre superieur dans la contiguité du trone. Paris 1887. Zitiert in Handbuch d. prakt. Chirurgie. 4. I. 